\title{
Designing of Capio-active, Lower Extremity and Brain Energized Full Body Exoskeleton with IoT Edge for Assisting Paralyzed Patients
}

\author{
Chirontan Bhuyan ${ }^{1}$, Anurag Gogoi ${ }^{2}$ \\ ${ }^{1}$ Mechanical Engineering Department, ${ }^{2}$ Civil Engineering Department \\ National Institute of Technology, \\ Silchar, Assam, India
}

\begin{abstract}
:
Exoskeleton can be defined as "wearable, external mechanical structure" whose objective is to reinforce or restore the physical performance of the person. The exoskeletons are placed on the user's body and can be classified into two categories:

- Passive: This kind of exoskeleton does not use any type of electrical power source. On the other hand, these are constituted with mechanisms as springs, dampers or high-pressure springs. It can be used for weight re-distribution or energy capture to support the users with the posture or motion.
\end{abstract}

- Active: unlike passive exoskeletons, active exoskeletons do use some type of actuator which in- creases human power. This actuator can be an electric motor, pneumatic muscles or hydraulic power.

Keywords: Exoskeleton, IoT, Paralyzed Patients, Assistive Technology, Lower Extremity Exoskeleton, Capio Active Upper Body Exoskeleton, Brain Energized Full Body Exoskeleton

\section{Introduction}

The most important problems for the production industry are that workers have to take sick leave because they have to recover from a physical disability that affects them to move and handle loading. This is called work-related musculo skeletal disorder (WMSD). However, WMSD does not occur just for handle loading, it can be also a consequence of high task repetition and awkward postures during work tasks.

The automotive sector is one of the industries most affected by the incidence of MSD. Several solutions have been tried to solve this condition in the automotive sector. One of these solutions is the vehicle rotation angle, which could allow to getting a variety of car postures during the assembly process.

Hence the need of exoskeleton comes into picture which are providing a realistic power enhancement for soldiers, firefighters, emergency personnel, and industrial workers increasing their physical potential and helping them and lifting heavy loads.

Thus, in simple terms these Exoskeletons are assistive wearable robotics connected to the body of a person, which aims to give mechanical power or mobility to the user.

The invention of the exoskeletons is credited to the Russian Nicholas Yagn (1890) who designed, what he called "Apparatus for facilitating walking, running and jumping", whose main objective was to reduce user fatigue. Exoskeletons- tons are starting to be implemented now in Industrial usage. Some of the automotive companies that are currently using exoskeletons in their assembly lines are Ford, Volkswagen or BMW. 
Figure: Apparatus for Walking, Running and Jumping
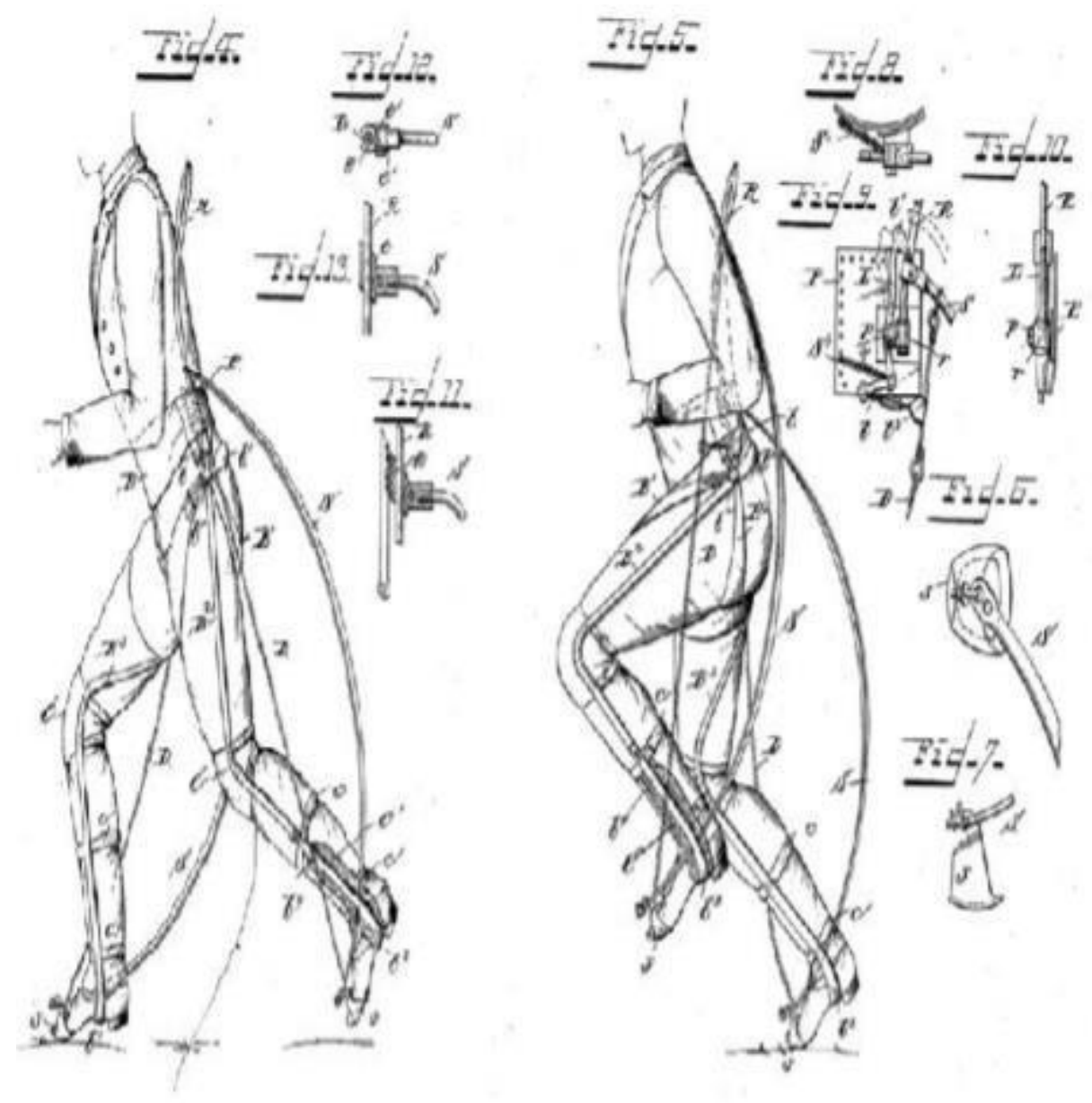

\section{Different Types of Exoskeletons}

EksoVest

EksoVest Exoskeletons is a passive upper body exoskeleton designed for those tasks that require an arm lift. The exoskeleton is attached to the lower back, the chest and the upper arm with the objective to transfer the load from the arms to the frame on the back.

Figure: EksoVest Exoskeleton

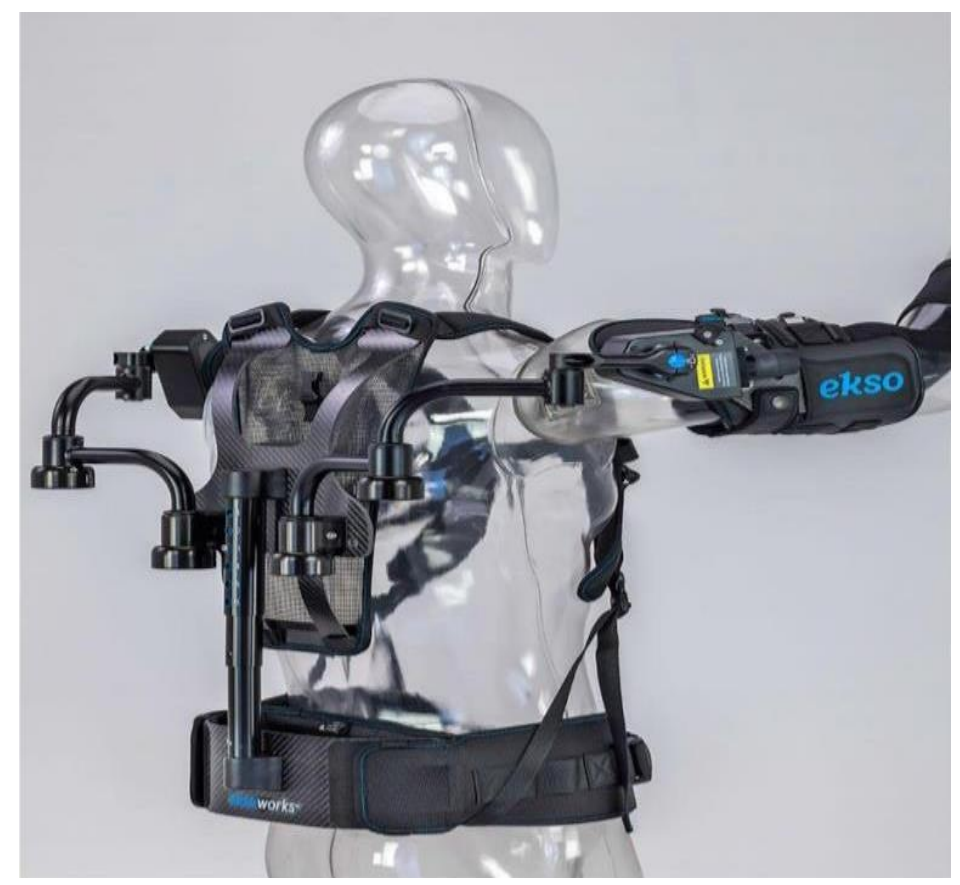




\section{Capio Exoskeleton}

The Capio exoskeleton is a human-machine interface that tracks the operator's movements.

Figure: Capio Active Exoskeleton

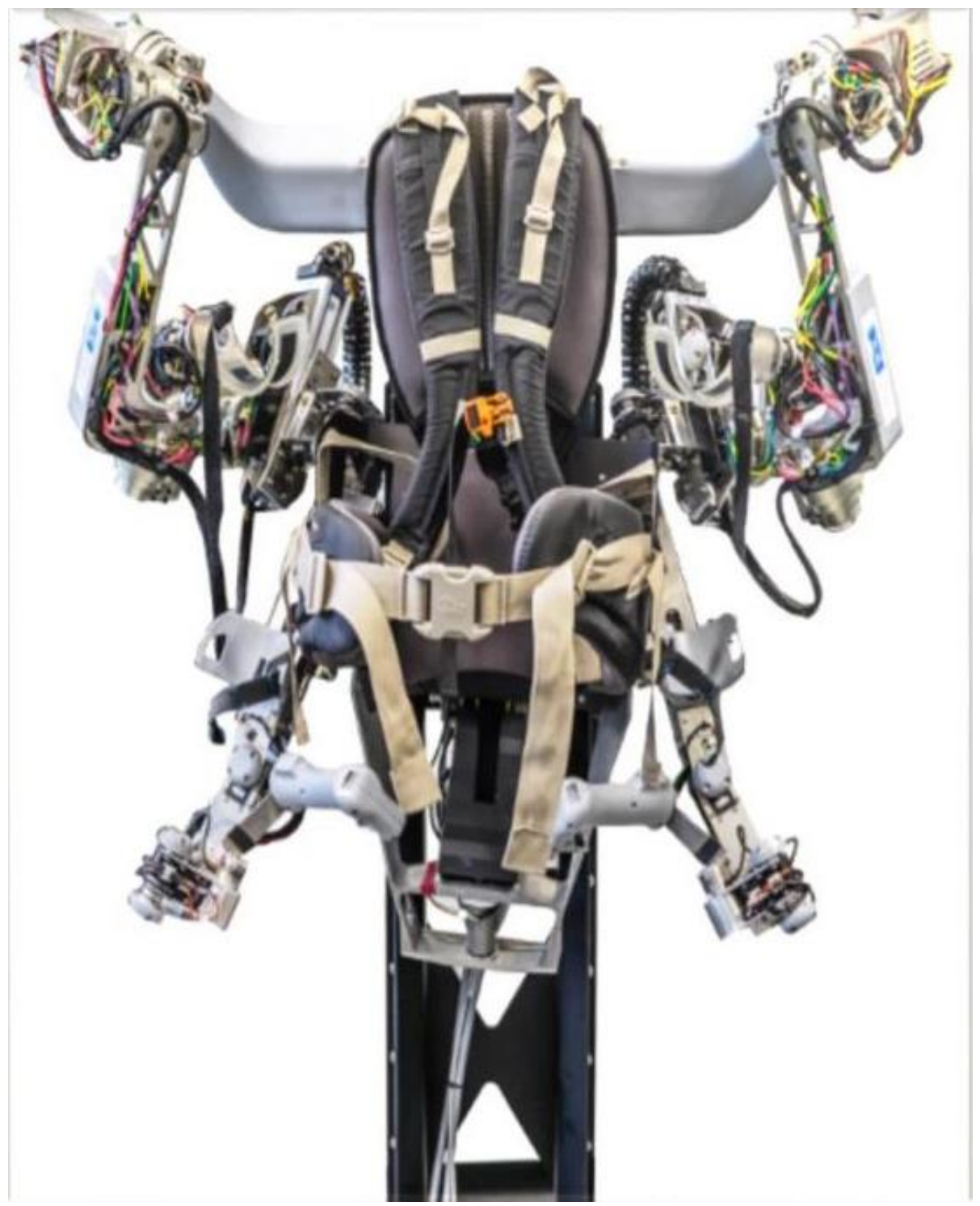

Table: Capio Exoskeleton Overview

\begin{tabular}{|l|l|}
\hline Feature & Value \\
\hline Active Degree of Freedom at the Back & $4[1]$ \\
\hline Active Degree of Freedom of One Arm & $8[1]$ \\
\hline Contact Points & $8[1]$ \\
\hline Current Consumption & $48 \mathrm{~A}$ \\
\hline Voltage & 12 and $48 \mathrm{~V}$ \\
\hline Total Weight & $24 \mathrm{Kg}$ \\
\hline
\end{tabular}

Multiple contact points at back and arms to the user enable a precise motion measurement and allow a specific force feedback. The combination of the kinematic configuration with 20 active serial elastic joints controlled by a rigid body dynamics algorithm provides mechanical transparency. The possibility to move the torso enhances the exoskeleton workspace. The Capio system is designed portable and light weight with an easy dressing procedure.

To test the functionality, a passive exoskeleton was built up as displacement study. With the passive exoskeleton, the joint angles can be measured and the corresponding velocities and accelerations can be calculated.

The kinematic structure includes two contact points at the back and three contact points at each arm. Advantage arises from the close arrangement between exoskeleton and user which enables a precise measurement and force feedback but enhances the required 
performance of the control algorithms and the kinematic.

Figure: Capio Exoskeleton CAD Kinematic Back Structure

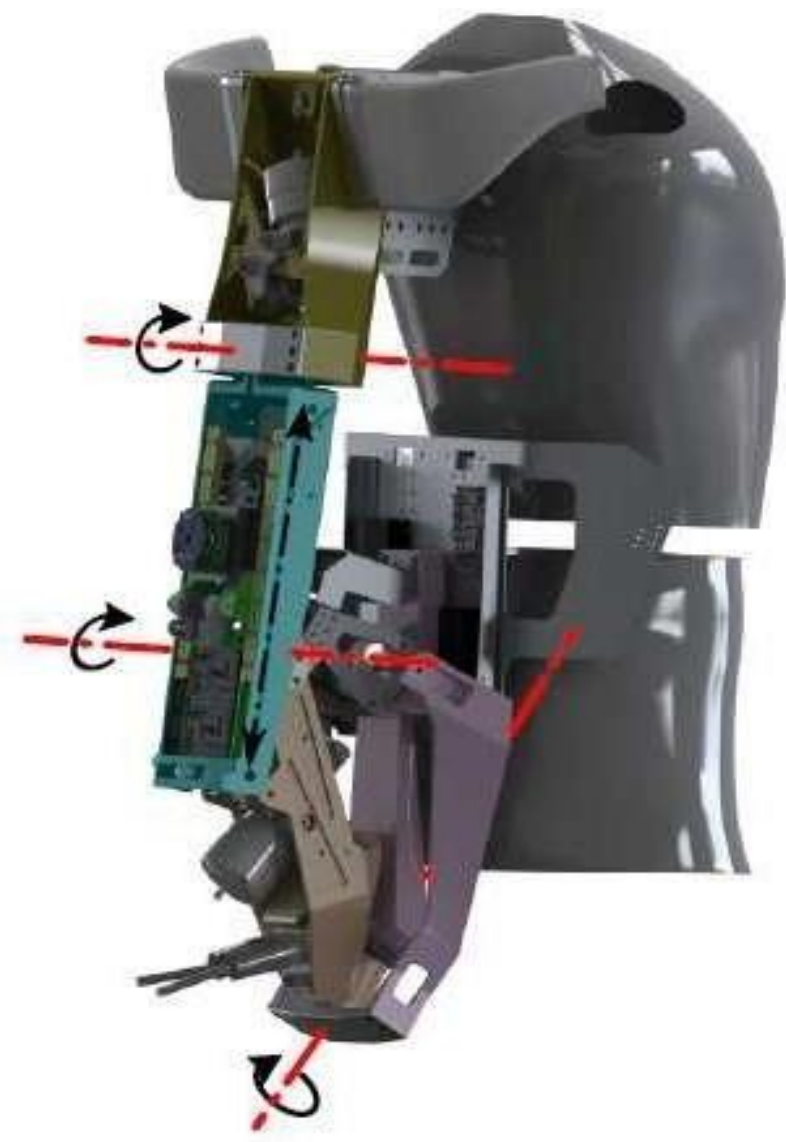

Four active degrees of freedom (DOF) realize the back movement with two adjustment possibilities.

The first joint allows the rotation of the back relative to the hip. The torso flexion and extension is achieved by the second joint in combination with a linear joint. The linear joint provides an extension of the structure, to compensate the larger movement radius of the structure. Eight active DOF are applied at each arm. Three drives represent the shoulder joint with a common intersection point. The elbow is simplified to a rotative joint in combination with a linear compensation joint. A passive joint connects the exoskeleton to the user's upper arm. To achieve an easy dressing procedure and improve the system's handling, an "Open Joint" concept is developed shown in below figure. The forearm pronation and supination are simplified to the longitudinal forearm axis. In this case a standard solution for an exoskeleton design is coaxial to this axis, often leading to a circular closed bearing design. In the "Open Joint" setup, the joint actuation and measuring axis is not coaxial to the pronation and supination axis but intersects with this axis in one center of rotation. This center of rotation is at the same time the intersection point of the wrist exoskeleton joints. Thereby, the misalignment of the pronation and supination of the exoskeleton is compensated. At the forearm Section of the exoskeleton the second contact is mounted.

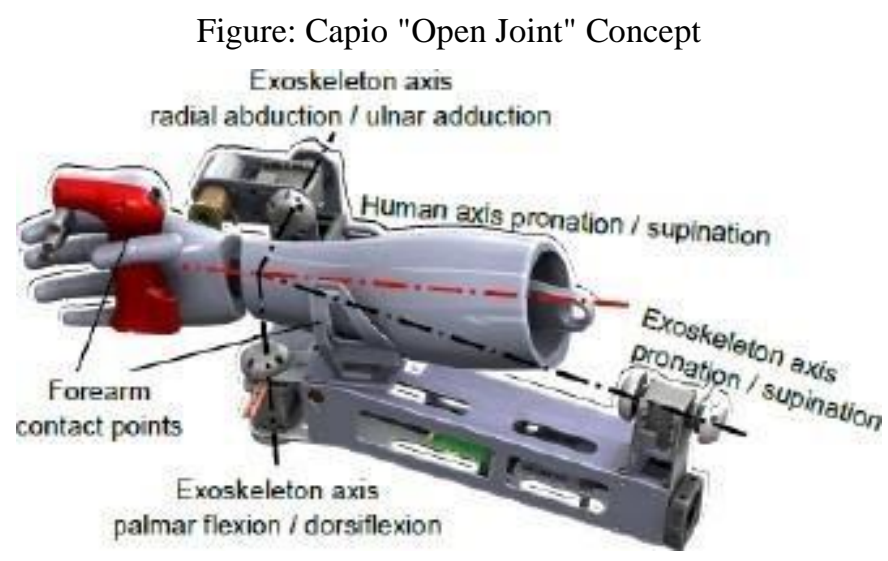

Lower Extremity Exoskeleton

These provide support to the legs, hips, and lower torso. 


\section{Mechatronic Design of Lower Limb Exoskeleton}

The design aims to be used as a walking support device focused on patients who suffer of partial lower body paralysis due to spine injuries or caused by a stroke. First, the mechanical design is presented and the results are validated through dynamical simulations performed in Autodesk Inventor and MATLAB. Second, a communication network design is proposed in order to establish a secure and fast data In between sensors, actuators, and microprocessors. Finally, patient-exoskeleton system interaction is presented and detailed. Movement generation is performed by means of digital signal processing techniques applied to electromyography (EMG) and electrocardiography (EEG) signals. Such interaction system design is treated and evaluated in MATLAB whose results are presented and explained. A proposal of real-time supervisory control is also presented as a part of the integration of every component of the exoskeleton.

Exoskeletons are designed to provide strength in gait and to transport heavy loads. There area also designs for assisting people with disorders in motion or elderly people. Gait rehabilitation is one of the greatest challenges for society in the upcoming years due to population aging and the increase of diseases affecting motion. A partial or total paralysis of one side of the body due to injuries in the motor centers of the brain is called Hemiplegia. Hemiplegia is a disorder that causes me half of the Iranian body to fail to perform its functions. This disorder is caused mainly due to stroke and in many cases, it is hereditary, Recovery from the stroke is difficult and the treatment is prolonged. Generally, an injury to the right side of the brain will cause a left-aided hemiplegia while an injury to the left side of the brain will cause a right- side dhemiplegia.

Wearable robotics is an area that provides solutions for such problems. A wearable robot extends, complements, or fully substitutes human function or empowers the Iranian limb where it is worn. These kinds of robots are classified according to the function they perform.

- Empowering Robotic Exoskeletons: These kinds of robots are known as extenders since they extend the strength of the human hand beyond its natural ability while maintaining human control of the robot.

- $\quad$ Robotic Orthotic: An orthoses maps the anatomy of a limb to gesture lust functions. The robotic counterpart of orthoses is robotic exoskeleton that complement the ability of the limbs. Exoskeletons are also capable of restoring handicapped functions.

- $\quad$ Prosthetic robots: These robots are devices that fully substitute lost limbs.

The below figures hows two examples of wearable robots. Scientific community differentiates exoskeletons from orthoses by defining the former as the devices that enhance physical capabilities of wholesome users, and the latter as the devices that assist persons with impairments in the limbs.

Inspite of their differences, both devices act in parallel with the limb. The applications of exoskeleton robots are varied, ranging from military applications to the medical field. In the medical field, exoskeletons in conjunction with rehabilitation therapies can assist patients with spinal cord injury, stroke and lower limb paralysis, caused by hemiplegia, to perform gait training.
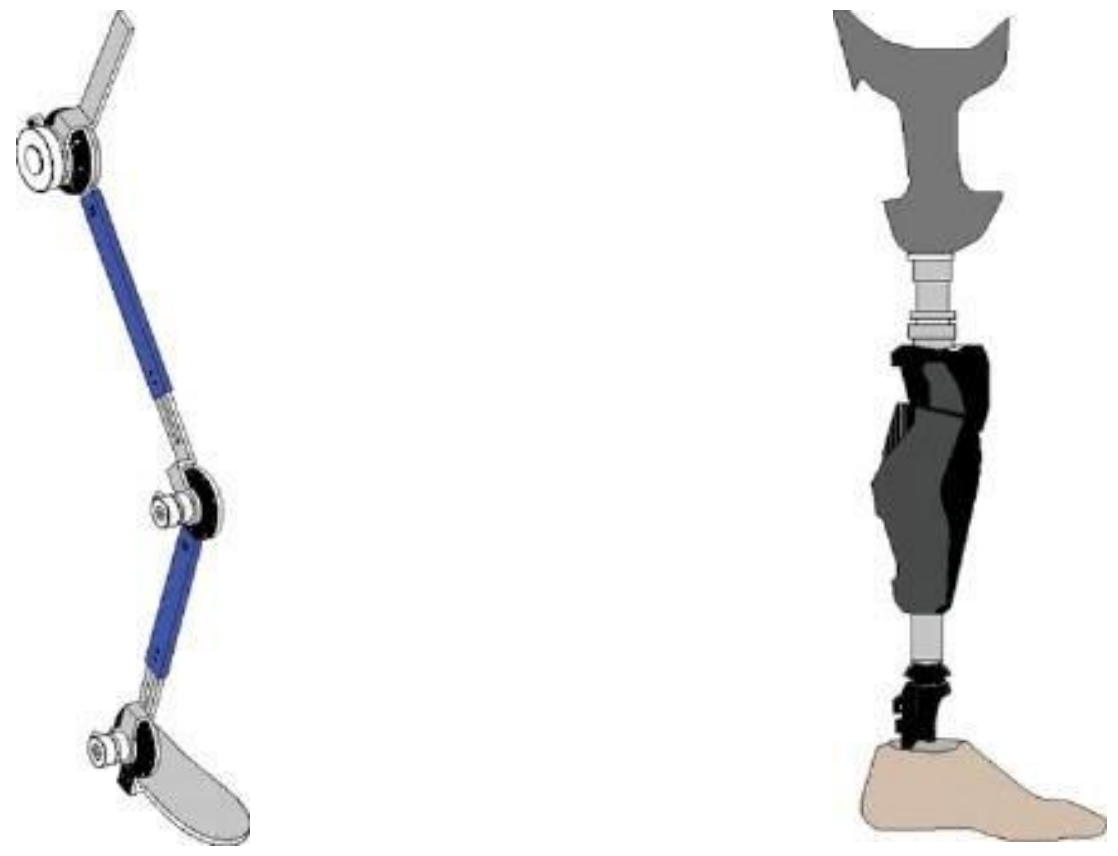

\section{Working and Architecture of Exoskeleton System}

The MPU performs the control of the exoskeleton using the data generated by SAUL. The SAUL implements the signal acquisition of the encoder and the strain gauges (if SAU is located at the ankle), the communication (transmission and reception), and the actuation of the control performed by the MPU.

The microcomputer used in the MPU is an embedded device with enough processing power to carry out the control task. Different 
options available commercially are Raspberry Pi, Beagle-Brine, Odroid, etc.

The use of communication devices is to reduce the wiring in the exoskeleton. The communication device in the MPU has two interfaces and in the SAU has one interface. The bus network topology is used for the communication. Each communication interface in the MPU operates one leg.

An Adaptive and Flexible Brain Energized Full Body Exoskeleton with IoT Edge for Assisting the Paralyzed Patients Majority of the existing exoskeletons have weight, flexibility, and adaptability constraints. Easy wearability and portability are other significant limitations experienced by current assistive exoskeleton-hated solutions for rehabilitation.

To overcome the current issues existing with exoskeletons, we propose an adaptive and flexible Brain Energized Full Body Exoskeleton (BFBE) fur assisting the paralyzed people. In the BFBE system, the brain signals captured by the EEG sensors are used for controlling the movements of the exoskeleton. The flexibility is incorporated into the system by a modular design approach. The BFBE system had a BC1 module, a Control Unit (CU), and a Body-Part Actuation Module (BAM). BCI module captures the brain signal and transforms it into a signal that can be used by the CU. The processing happens at the edge, thus reducing delay in decision making, and the system is further integrated with an IoT module that helps to send air alert message to multiple caregivers in case of an emergency. The system is non-invasive, and the fabricated EEG sensor is used to collect the signals from the scalp. An instrumentation amplifier is used to enhance the strength of the obtained signals. The output signal from the amplifier is subjected to filtering and pre-processing. The signals are generated for different basic human actions (sitting, standing, sleeping) and then after the pre-processing is stored in a database. When the paralyzed person has an intention to make a particular movement, the microcontroller in the CU uses this data base and produces the signal fur activating the particular body part. The generated EEG pattern of the person is mapped into the corresponding action. The BAM then uses the motor driver circuit to pass the activation signal to the corresponding part of the body. The potential energy harvesting is used in the system to solve the power issues related to the exoskeleton. The stability in the gait cycle is ensured by using adaptive sensory feed-back.

\section{System Architecture}

The architecture of the Brain Energized Full Body Exoskeleton (BFBE) system is shown in below figure. The BFBE system has three major modules (1) BC1 module, (2) Control Unit, and (3) Body-Part Actuation Module. The primary function of the BC1 module is to collect the EEG signals from the scalp and then convert it into a form ease. The BFBE has a total of 15 degrees of freedom that can be used by the CU. We have fabricated freedom spread across different joints of the a sixteen-electrode based EEG sensor, which is used in the proposed system for collecting the signals and also for analyzing the brain activity. For removing the high-frequency noise, we use a bandpass. Walsh Hadamard Transform (WHT) is then used to transform the signals into the frequency.

Figure: Sitting Posture of BFBE (3D Model)

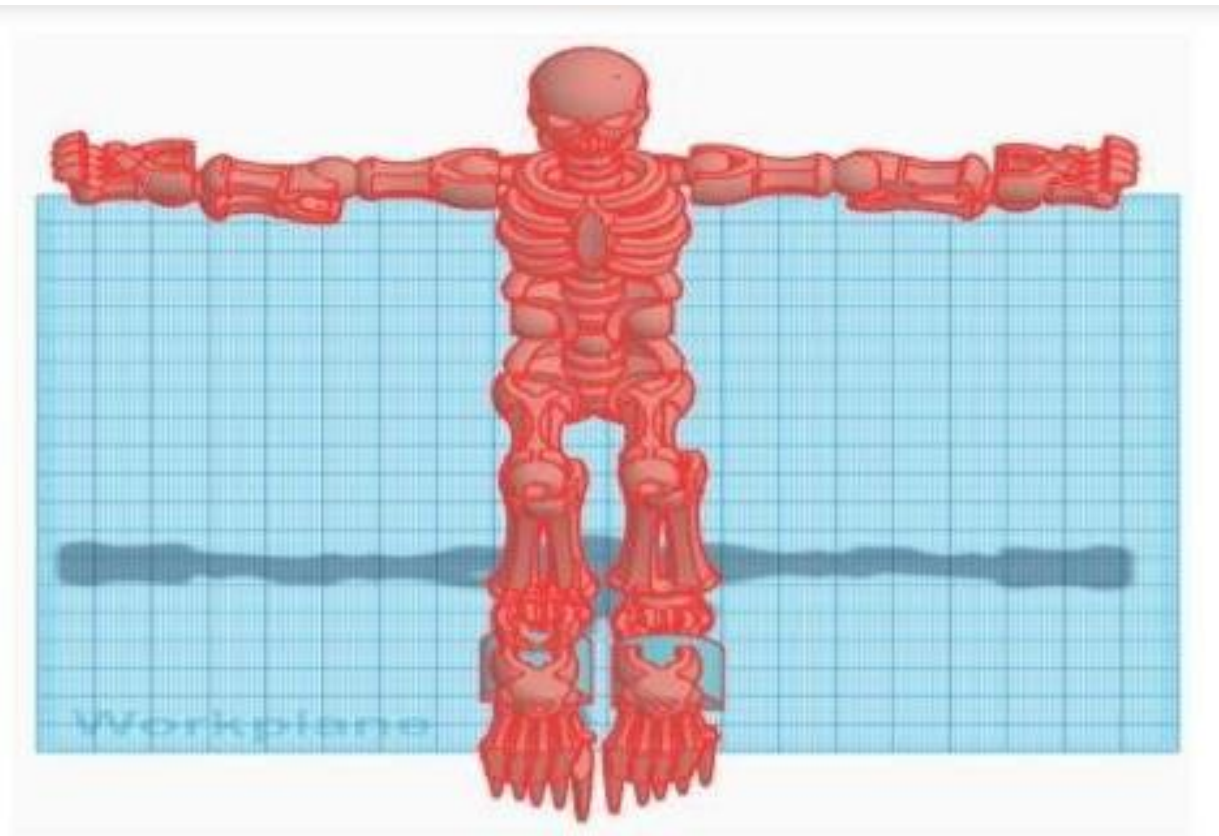


EEG Sensor Manufactured with 16 Electrodes
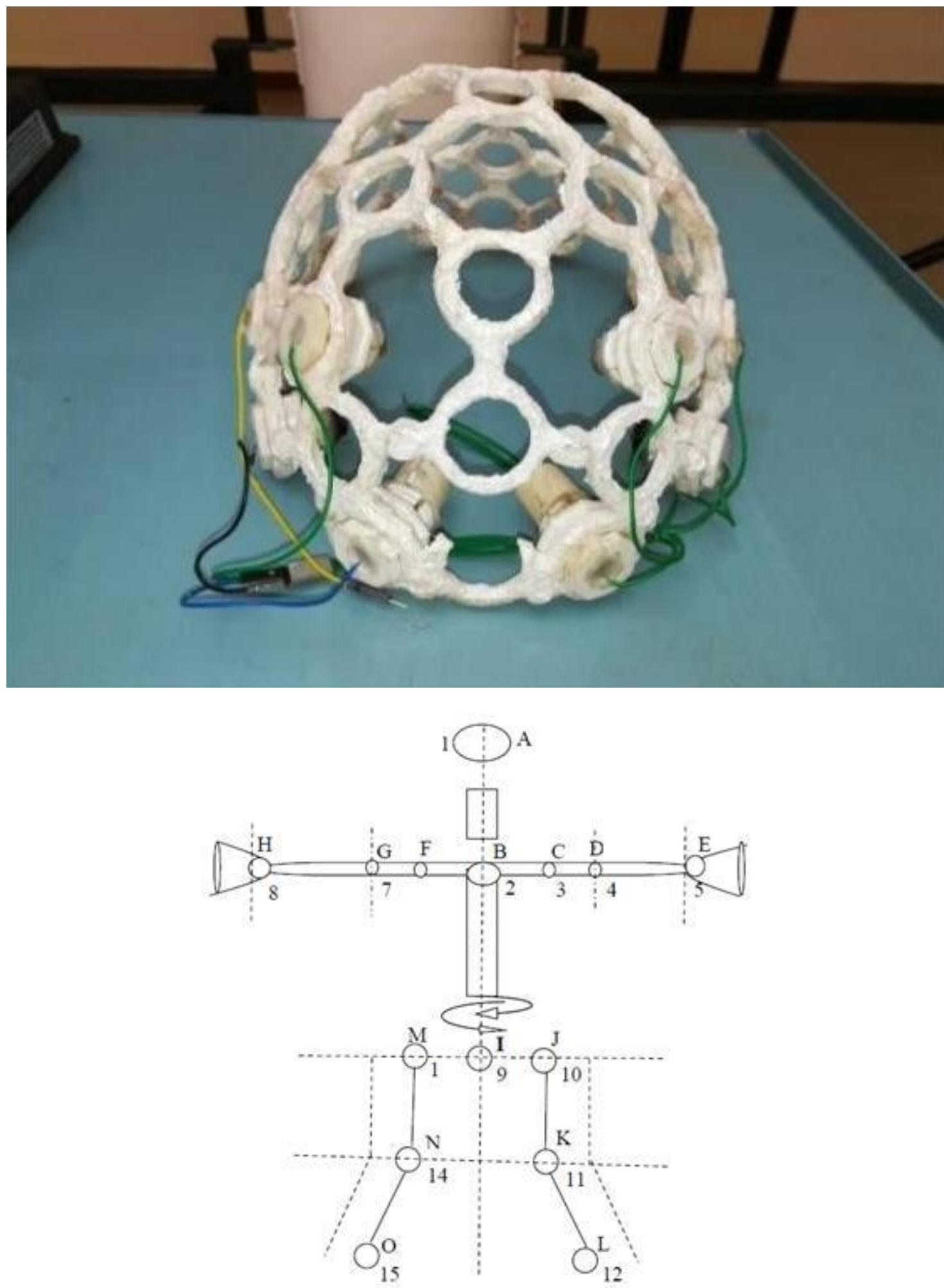

The signals are further transformed into digital form and provided to the Arduino Mega, which transmits the signal via Bluetooth to the Control Unit. The signals are generated for different basic human actions (sitting, standing, sleeping) and then after the pre-processing is stored in a database. When the paralyzed person has an intention to make a particular movement, the micro-controller in the $\mathrm{CU}$ read this database and produces the signal for activating the particular part of the body. The generated EEG pattern of the person is mapped into the corresponding action. The BAM then uses the motor driver circuit to pass the activation signal to the corresponding part of the body. The potential energy harvesting is used in the system to solve the power issues related to the exoskeleton. The stability in the gait cycle is ensured by using adaptive sensory feedback. The feedback to the CU is provided using a multi-level sensing technique so that corrections can be made in the process. Required corrections for producing the actuation signals are done by the microcontroller this feedback and thus improving the decision- making accuracy. An accelerometer is used on the backside for detecting accidental falls. The pmceasing happens at the edge, thus reducing delay in decision making, and the system is further integrated with an IoT module 
that helps to send an alert message to multiple caregivers in case of an emergency. If the measured tilt passes a particular threshold, a similar emergency message will be given to the caregiver via a wireless transmitter. The secure communication is ensured between paralyzed persons and caregivers using a double encrypted NTSA algorithm. The material used for the development is carbon fiber, so that it can easily replicate various body movements.

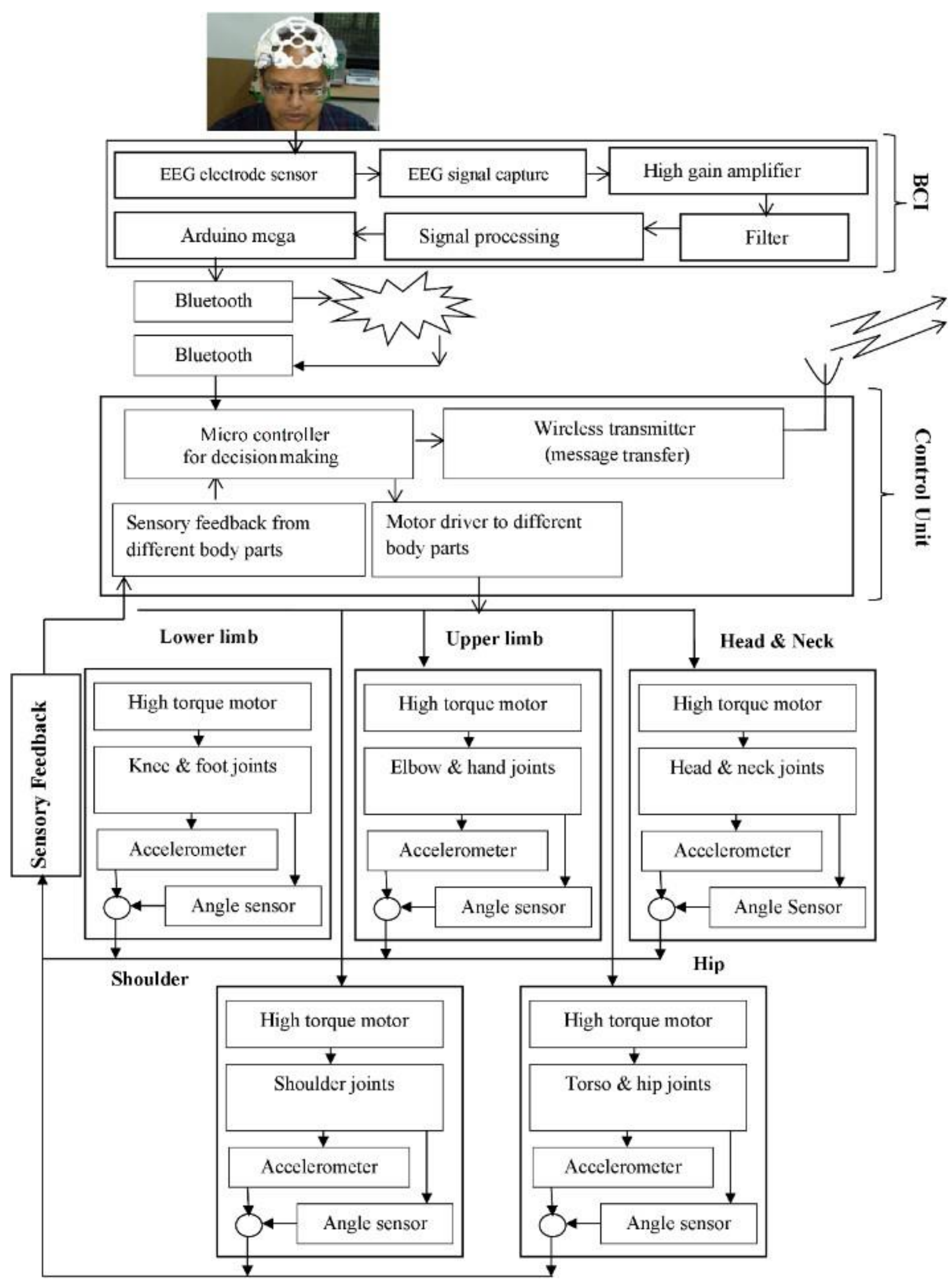




\section{Advantages of Exoskeletons}

- The disabled people are finally able to overcome their disabilities.

- The exoskeleton amplifies the strength and endurance of the user.

- The quality of life improves.

- This industry provides an expanding market and newer jobs.

- The exoskeleton provides a support to the body while doing work.

- Above all, exoskeletons are helpful for soldiers in war when there comes a conflict between countries.

\section{Disadvantages of Exoskeletons}

- The weight of the exoskeleton can put a burden on the user's body.

- These exoskeletons are quite expensive.

- Manufacturing of specialized parts is required.

- They cannot stretch or expand.

- Special modification is required for gaseous exchange and sensory pick up.

- It is a major restriction on growth.

- It needs to be shed at regular intervals.

Challenges that will be Faced in Adaption for Exoskeleton

- The power supply integration with the exoskeleton is complex.

- The skeleton material decision should be done to avoid damages.

- The decision of selecting the actuators has to be made for proper functioning.

- The most difficult task for an exoskeleton is to replicate motions of ball joint using a series of external single-axis hinge points, thus limiting the flexibility of the user.

- $\quad$ The exoskeleton should be able to adapt easily to user size variations

Despite the advantages and disadvantages, there are major issues associated with the kinematic compatibility of exoskeletons with human arm, which makes design of prosthetic devices challenging. In addition to design issues, development of effective control algorithms appropriate for rehabilitation goals has been a major challenge, partially due to the aforementioned reasons many exoskeleton systems are bulky, very complex to operate, costly and heavy which limit widespread use of them.

\section{References}

[1] https://exoskeletonreport.com/what-is-an- exoskeleton/

[2] https://jneuroengrehab.biomedcentral.com/ar ticles/10.1186/s12984-020-00663-9

[3] https://en.wikipedia.org/wiki/Exoskeleton

[4] https://www.slideshare.net/ayushisrivastava1 46/ame527final21

[5] https://www.slideshare.net/ManjuSajjanar/ex oskeleton-arms?qid=31a9cadb-46a5-4d9c- aac1b1d $997 \mathrm{~d} 2 \mathrm{~d} 604 \& \mathrm{v}=\& \mathrm{~b}=\&$ from_search $=2$

[6] https://www.slideshare.net/simplyAloki/desi gn-of-lower-limb-exoskeleton-65893227

[7] https://www.lockheedmartin.com/en- us/products/exoskeleton- technologies/military.html 